\section{Problemas éticos y de salud planteados por la reciente epidemia de Ébola: ¿Qué debemos aprender?}

\author{
JUAN PABLO BECA ${ }^{1}$, SOFÍA P. SALAS ${ }^{2}$
}

\section{Ethical and health issues posed by the recent Ebola epidemic: What should we learn?}

The recent Ebola epidemic that affected several countries in Africa, with very high mortality and a pandemic threat, posed problems of justice, public health, prevention, treatment and research, each of which has relevant ethical issues. Despite severe initial difficulties, an effective international response was achieved, whose outcome has left significant teachings to be considered in order to deal with future epidemics or pandemics. In this article, the authors analyze the main problems faced during the Ebola epidemic, including the unequal distribution of health resources between countries, the need for international collaboration, the requirement for a review of the ethical standards of clinical trials in emergencies, and the necessity of an organized global system of prevention and timely response to these outbreaks. Authors conclude that at the present time health is a global issue without borders, that insufficient healthcare resources in some countries poses risks and affects all countries and that the confrontation of the threats of epidemics requires a solution based in universal solidarity. At the same time, a moral duty to investigate should be acknowledged, seeking a balance between sense of urgency, scientific rigor and involvement of local communities.

(Rev Med Chile 2016; 144: 371-376).

Key words: Ebola virus; Ethics, research; Public Health; Social Justice.
${ }^{1}$ Centro de Bioética. Facultad de Medicina Clínica Alemana Universidad del Desarrollo. Santiago, Chile.

2Programa de Ética y Políticas Públicas en Reproducción Humana, Facultad de Medicina, Universidad Diego Portales. Santiago, Chile.

Conflictos de interés: Ninguno que declarar. Este trabajo no obtuvo financiamiento.

Recibido el 2 de marzo de 2016, aceptado el 15 de marzo de 2016.

Correspondencia a: Juan Pablo Beca

Av. Las Condes 12438,

Lo Barnechea.

Teléfono: 223279157

jpbeca@udd.cl
É bola, pequeño río de la República Democrática del Congo (antiguo Zaire), dio nombre al virus que genera una enfermedad hemorrágica viral con una alta tasa de mortalidad que ha desencadenado brotes epidémicos descritos desde 1976 en África ${ }^{1}$. El reservorio del virus en la naturaleza serían murciélagos de la fruta (familia Pteropodiae) y éste se transmite al hombre por contacto directo o indirecto con estos murciélagos u otros mamíferos infectados, como monos y antílopes. Una vez que se transmite al humano se inicia un ciclo de transmisión interhumana, en que las personas enfermas contagian el virus a sus contactos cercanos a través de su sangre y otros fluidos corporales, incluyendo transmisión sexual. Después de 40 años del brote inicial, la investi- gación sobre esta trágica enfermedad había sido escasa y lenta, como ocurre con muchas enfermedades que afectan de manera muy predominante al tercer mundo.

Las epidemias de Ébola entre 1976 y 2012 fueron de decenas o centenas de casos (Figura 1) que se controlaron por la evolución de sus propios ciclos epidémicos ${ }^{2}$. A pesar de su alta mortalidad, no se generó alarma mundial y la investigación sobre el tema fue escasa y en parte motivada por tratarse de una infección viral que potencialmente podría ser un arma biológica. El reciente brote, con más de 28.000 casos y más de 11.000 muertes, se inició en diciembre de 2013 en Guinea y se extendió de manera rápida a Sierra Leona y Liberia a inicios de 2014. Luego se diseminó en forma 


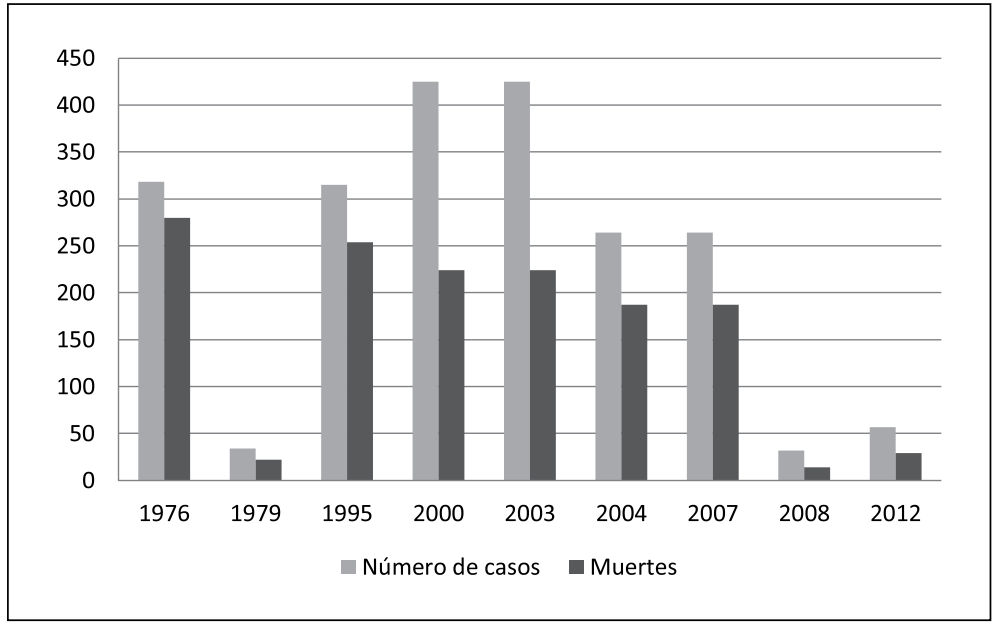

Figura 1. Número de casos y de muertes registradas en las epidemias de Ébola entre los años 1976 y 2012. Datos tomados de información de OMS (Ref 2). mucho más intensa debido a las características actuales de los países afectados: sistemas de salud muy precarios, alta movilidad de la población, pobreza, hacinamiento y características culturales de los funerales. Además, la infección llegó a ciudades donde la transmisión se multiplicaba en forma descontrolada ${ }^{3}$.

Ocho meses después, en agosto de 2014, se decretó alarma mundial después que dos norteamericanos se contagiaron y fueron trasladados a hospitales en Estados Unidos de Norteamérica (EE.UU. ${ }^{4}$. El principal problema era el temor de una pandemia y cómo contener la expansión de la epidemia con la evidencia de que no era posible frenarla con los recursos de los países afectados, sin tener antivirales ni vacunas de eficacia y seguridad demostrada. Se reconoció la existencia de un problema de justicia distributiva internacional y la necesidad de avanzar hacia una solidaridad internacional y cosmopolita ${ }^{5}$.

Surgieron numerosas repuestas para responder a la emergencia, desde la Organización Mundial de la Salud (OMS) y desde gobiernos europeos y de América del Norte. Se reconoció también la necesidad de combinar la respuesta social y comunitaria con la organización urgente de generar conocimiento mediante sólidos proyectos de investigación, definiendo así un concepto de "deber moral de investigar"6. La realidad de recursos sanitarios en estos países hacía muy difícil el control de la epidemia, con lo cual se generó mayor temor, desconfianza de las comunidades locales en sus instituciones y en su personal, y también en las intervenciones de expertos extranjeros ${ }^{7,8}$. Los recursos curativos existentes en ese momento estaban todavía en fases iniciales de investigación con antivirales o anticuerpos monoclonales que aún no habían sido usados en seres humanos ${ }^{9,12}$. En estas condiciones era necesario intervenir conjuntamente en capacitar personal de salud, establecer recursos diagnósticos y de medidas generales de cuidado y compensación de pacientes, tratamiento del shock hipovolémico con sus consecuencias, así como investigar aplicando rigurosidad científica $y$ ética en los respectivos proyectos ${ }^{13}$. Todo lo anterior, con la participación de la comunidad de los tres países afectados, no sólo por razones de justicia sino como condición indispensable para poder realizar estas intervenciones ${ }^{14}$.

Las respuestas, aunque tardías como ya se mencionó, se implementaron en forma acelerada de parte de OMS y de los países del hemisferio norte, convocando a especialistas en salud pública, investigación, epidemiología y bioética. OMS convocó a un panel multidisciplinario de expertos, que propuso criterios generales para las respectivas intervenciones ${ }^{6}$. Cabe destacar la participación de Médicos Sin Fronteras, de universidades y de la industria farmacéutica, cada uno en sus respectivos campos de acción. En los países no afectados se comprendió el riesgo de contagios internacionales o de una pandemia, de manera tal que se trabajó en capacitar al personal de salud y en implementar los recursos necesarios para evitar contagio entre enfermos o sospechosos de contagio y el personal o las familias de los mismos. 
A pesar de todo el esfuerzo, esta epidemia de Ébola, como es sabido, fue la mayor de la historia con 28.627 casos demostrados y 11.315 fallecidos en los años 2014-2015.

Finalmente se logró decretar, el 14 de enero de 2016, el término de esta epidemia, después de 42 días sin nuevos casos en Liberia. Previamente se había decretado libre de Ébola a Sierra Leona, en noviembre, y a Guinea el 29 de diciembre de $2015^{15}$. Es digno de celebrar este logro alcanzado en un plazo breve de tiempo. Sin embargo, si se piensa en el costo en enfermos, sufrimiento, vidas humanas, detención de las economías, esfuerzos humanos y económicos de tantos países, se puede llegar a la conclusión de que el costo y el riesgo actual de una epidemia superan los riesgos de catástrofes naturales y de muchas guerras. Por esta razón resulta necesario recopilar la experiencia y lo que todos debemos aprender de ella.

En plazos relativamente breves se definieron formas de control de contagios y de expansión de la epidemia, se capacitó a miles de trabajadores de la salud y a miembros de la comunidad en más de 100 países, se iniciaron proyectos de investigación de métodos diagnósticos, fármacos antivirales y vacunas con sus respectivos fundamentos científicos y éticos, y se logró recuperar confianzas y trabajar con la población de los países afectados. Así se llegó a contar con métodos rápidos de confirmación diagnóstica, fármacos antivirales e inmunológicos en estudio en fase II, y vacunas ya en ensayos clínicos fase III, con stock disponible ante nuevos riesgos epidémicos ${ }^{16}$. Cabe hacer notar que estos avances en investigación se lograron en 12 meses, siendo la duración habitual para estos estudios de un promedio de 10 años.

La reciente epidemia de Ébola se constituyó en una crisis humanitaria que ocupó la atención de los medios de comunicación, gobiernos y organismos internacionales, que movilizaron enormes recursos humanos y económicos ante un riesgo global ${ }^{17}$. De esta manera resulta importante plantearse la pregunta de qué hemos aprendido de esta epidemia y sus graves consecuencias, para poder enfrentar nuevos riesgos de epidemias que amenazan a los países y sociedades contemporáneas.

La respuesta mundial a este grave y reciente brote significó un interés no sólo de gobiernos y organizaciones internacionales sino de toda la comunidad científica. Un signo revelador al respecto es el aumento de publicaciones; por ejemplo, en
PubMed el número de referencias sobre Ébola aumentó de 265 entre 2012 y 2013 a 2.982 referencias entre 2014 y 2015 . Ha quedado claramente demostrado que la gravedad de casos disminuye fundamentalmente por medidas generales, hidratación oral y parenteral, y óptimo control de síntomas y complicaciones, lo cual requiere niveles de infraestructura hospitalaria que eran gravemente insuficientes en los países afectados. Por otra parte, la transmisión de la enfermedad también se relaciona con la infraestructura y recursos de atención de salud. A raíz de las investigaciones y ensayos clínicos realizados con urgencia, incluyendo el uso de fármacos o vacunas en seres humanos, hoy se cuenta con grandes avances. Se dispone de tratamientos potenciales en base a productos sanguíneos, terapias inmunológicas y antivirales en fases avanzadas de investigación, junto a dos vacunas ya en fase de demostrar su seguridad ${ }^{2,17}$. Las investigaciones mencionadas se concretaron gracias al consenso logrado en cuanto a la flexibilización de los requisitos éticos de la investigación frente a emergencias graves y extensas como la que se vivía. Así fue como se utilizaron fármacos experimentales con criterio compasivo, pero aplicando para este efecto estrictos protocolos de investigación ${ }^{6,18}$.

No obstante lo anterior, se han reconocido diferentes problemas sociales y perspectivas éticas en el desarrollo de la epidemia, en las respuestas de los diferentes países, de las organizaciones internacionales, de la industria y de la comunidad académica $^{11,19-22}$.

Una de las respuestas más destacadas ha sido la toma de conciencia de que, a pesar de la historia de epidemias antiguas y recientes (VIH, H1N1, SARS y MERS), el mundo se encuentra aún mal preparado para enfrentar crisis de enfermedades infecto-contagiosas. Hubo reacción lenta y tardía, sistemas locales de salud completamente insuficientes y colapsados, incoordinaciones a todo nivel, falta de tests diagnósticos rápidos y de equipamiento de protección del personal sanitario, y escasa investigación de tratamientos y vacunas. En este contexto se creó la Commission on a Global Health Risk Framework for the Future, bajo el liderazgo de la Academia Nacional de Medicina de EE.UU. ${ }^{23}$. En reuniones de científicos, representantes de gobiernos, industria, organizaciones sociales, Naciones Unidas, OMS y otros, se destacó que las epidemias o pandemias constituyen 
mayor amenaza de pérdida de vidas humanas que la guerra, terrorismo o desastres naturales. Sin embargo, no se tiene suficiente conciencia de este riesgo ni se destinan los recursos necesarios para la investigación y para soluciones oportunas. Concluye esta comisión que las pandemias son la parte más descuidada de la seguridad global, y que la seguridad global en salud constituye un bien público: la mayor seguridad de cada uno de nosotros depende de la seguridad de todos. Confirma esta mirada lo que se está viviendo en este momento con la expansión del contagio por virus Zika, transmitido en países americanos de clima tropical por un mosquito del género Aedes aegypti proveniente de bosques de Uganda.

\section{Problemas éticos en la investigación en Ébola}

Además de los problemas sanitarios y humanitarios ya descritos, la epidemia de Ébola trajo consigo una importante discusión respecto de cómo realizar investigación médica en situación de epidemia, especialmente en países en vías de desarrollo. En primer lugar, fue evidente la necesidad de promover investigación multidisciplinaria, puesto que la interacción entre los investigadores biomédicos y los de ciencias sociales mostró ser crucial para comprender y negociar de manera apropiada, respetando los valores culturales que estaban en juego. Junto a esto, también fue indispensable promover la colaboración entre investigadores de naciones desarrolladas y aquellos de países en vías de desarrollo, de tal modo de hacer frente a la emergencia sanitaria venciendo las naturales resistencias iniciales. Otro punto de interés fue reflexionar sobre la forma de repartir los beneficios de la investigación para los participantes de los estudios clínicos y las poblaciones locales, que se consideran como co-productores del conocimiento, estableciendo mecanismos para poder compartir rápidamente los resultados científicos tanto a nivel local como a nivel internacional, durante los brotes y las crisis de salud pública ${ }^{24}$. Un segundo aspecto relevante en ética de la investigación fue el uso de terapias experimentales. En el comienzo de este brote, estaba en desarrollo un suero inmunológico (zMapp), que había sido probado solamente en monos. Debido a la emergencia sanitaria, se consideró que era éticamente justificado hacer las primeras pruebas en huma- nos, sin recurrir a las clásicas fases de desarrollo de un nuevo producto. Los expertos de bioética consultados por la OMS consideraron que dada la emergencia era un imperativo ético usarlos, pero que su uso debía ser regulado de tal modo de permitir obtener resultados válidos que pudiesen servir para futuros pacientes, equilibrando el rigor científico de los proyectos con la aceptabilidad local en circunstancias tan especiales, aun cuando esto se hiciese sin cumplir con el estándar de oro de ensayos clínicos asignados al azar, sino que más bien adaptados a la emergencia sanitaria ${ }^{25,26}$. Así, se desarrolló el concepto de "ensayo clínico aleatorio adaptable", como una buena alternativa para permitir obtener resultados válidos y en corto tiempo ${ }^{27}$.

\section{A modo de conclusión}

La emergencia sanitaria producida por la rápida expansión del virus Ébola deja en evidencia que la salud es un tema global que no reconoce fronteras, por lo cual es necesario estar preparados de manera adecuada para enfrentar futuras epidemias. Confirma lo anterior la epidemia del virus Zika, que actualmente está afectando a varios países de la región ${ }^{28}$.

Dado que existen condiciones socioeconómicas que facilitan que estas enfermedades infecciosas se transmitan con prontitud en zonas menos aventajadas, que no poseen los recursos sanitarios ni humanos para hacerles frente, es necesario actuar de manera solidaria, utilizando recursos y capacidad tecnológica que necesitan ser provistos por países con más recursos. En este sentido existe un problema de justicia distributiva, que requiere como respuesta una solidaridad internacional y sin fronteras, para así lograr un adecuado control, prevención y tratamiento de los enfermos, como expresión de un derecho humano básico que no puede depender de los recursos insuficientes de un país en particular. Por último, dada la imperiosa necesidad de obtener respuestas eficaces de forma urgente, se hace necesario flexibilizar la forma de llevar a cabo los protocolos de investigación ante emergencias epidemiológicas o naturales, con diseños que se adapten a las realidades particulares. Creemos firmemente que existe un deber moral de investigar, de tal modo que de este tipo de situaciones epidemiológicas se puedan obtener resultados 
generalizables. Concordando con esta mirada, un reciente reporte de un panel de expertos convocados por el Harvard Global Health Institute y el London School of Hygiene \& Tropical Medicine identificó cuatro áreas temáticas que era necesario tener en cuenta ante futuras pandemias ${ }^{29}$. En primer término, se requiere prevenir brotes de enfermedades graves, para lo cual se necesita apoyo de la comunidad internacional para mejorar las capacidades instaladas; en segundo lugar, es necesario dar respuesta oportuna a brotes de enfermedades serias, con la participación de la Organización Mundial de la Salud; en tercer lugar, en el área de investigación, es necesario producir y compartir datos, conocimiento y tecnología, lo que requiere un trabajo multidisciplinario y normas claras para asegurar acceso a los beneficios de la investigación; $y$ en cuarto lugar, es necesario tener un sistema global organizado para la prevención y respuesta ante estos brotes.

\section{Referencias}

1. Report of an International Commission. Ebola haemorrhagic fever in Zaire, 1976. Bull World Health Organ 1978; 56 (2): 271-93.

2. WHO Fact sheet 103. 2016 January. Ebola virus disease. Disponible en http://www.who.int/mediacentre/factsheets/fs103/en/.

3. Kanapathipillai R. Ebola virus disease-current knowledge. N Engl J Med 2014; 371 (13): e18.

4. Hawkes N. Ebola outbreak is a public health emergency of international concern. Br Med J 2014; 349: g5089.

5. Prainsack B, Buyx A. Solidarity in contemporary bioethics-towards a new approach. Bioethics 2012; 26 (7): 343-50.

6. WHO Advisory Panel for Ebola Outbreak. Ethical considerations for use of unregistered interventions for Ebola viral disease. August 17, 2014. http://apps.who. int/iris/bitstream/10665/130997/1/WHO_HIS_KER_ GHE_14.1_eng.pdf?ua=1.

7. Chowell G, Nishiura H. Transmission dynamics and control of Ebola virus disease (EVD): a review. BMC Med 2014; 12 (1): 196.

8. Check Hayden E. Ebola obstructs malaria control. Nature 2014; 514 (7520): 15-6.

9. Choi JH, Croyle MA. Emerging targets and novel approaches to Ebola virus prophylaxis and treatment. Bio Drugs 2013; 27 (6): 565-83.

10. Qiu X, Wong G, Audet J, Bello A, Fernando L, Alimonti
JB, et al. Reversion of advanced Ebola virus disease in nonhuman primates with ZMapp. Nature 2014; 514 (7520): 47-53.

11. Rid A, Emanuel EJ. Ethical considerations of experimental interventions in the Ebola outbreak. Lancet 2014; 384 (9957): 1896-9.

12. Gulland A. Ebola outbreak in west Africa is officially over. BMJ 2016; 352: 243.

13. WHO. Latest Ebola outbreak over in Liberia; West Africa is at zero, but new flare-ups are likely to occur. Jan 14, 2016. http://www.who.int/mediacentre/news/ releases/2016/ebola-zero-liberia/en/.

14. Calain P, Poncin M. Reaching out to Ebola victims: Coercion, persuasion or an appeal for self sacrifice? Social Science \& Medicine 2015; 147: 126-33.

15. WHO updates, October 2015. Ebola vaccines, therapies, and diagnostics. http://www.who.int/medicines/ emp_ebola_q_as/en/.

16. Heron RJL. Ebola-what have we learned from the recent outbreak? Occup Med 2016; 66: 4-7.

17. Kennedy SB, Neaton JD, Lane HC, Kieh MW, Massaquoi MB, Touchette NA, et al. Implementation of an Ebola virus disease vaccine clinical trial during the Ebola epidemic in Liberia: Design, procedures and challenges. Clin Trials 2016; 13 (1): 49-56.

18. Mendoza EJ, Qiu X, Kobinger GP. Progression of Ebola Therapeutics During the 2014-2015 Outbreak. Trends in Molecular Medicine 2016; 22 (2): 164-73.

19. Beca JP, Valdivieso F. Epidemia de Ébola: una mirada desde la infectología y de la Bioética. Boletín Complutense 2014; 11-5.

20. Gericke C. Ebola and ethics: autopsy of a failure. BMJ 2015; 350: h2105.

21. Mondragon NI, Gil de Montes L, Valencia J. Understanding an Ebola outbreak: Social representations of emerging infectious diseases. J Health Psychol 2016, Jan 7. DOI: $10.117 / 1359105315620294$.

22. Dodd LE. Clinical trials during the Ebola crisis. Clin Trials 2016; 13 (1): 5.

23. Sands P, Mundaca-Shah C, Dzau VJ. The Neglected Dimension of Global Security-A Framework for Countering Infectious-Disease Crises. N Engl Med 2016; DOI:10.1056/NEJMsr1600236.

24. Fenet $\mathrm{S}$. Towards a sustainable sharing of data \& samples collected during trials effectuated in resource-limited countries. Workshop organized by the Inserm Ethical Committee, Fondation Mérieux \& the Global Forum on Bioethics in Research (GFBR), 5 noviembre 2015. Disponible en http://www.fondation-merieux.org/towards-better-sharing-of-data-and-samples-collected-during-trials-in-resource-limited-coun- 
tries-2015-background. (Consultado el 1 de marzo de 2016).

25. Adebamowo C, Bah-Sow O, Binka F, Bruzzone R, Caplan A, Delfraissy JF, et al. Randomised controlled trials for Ebola: practical and ethical issues. Lancet 2014; 384 (9952): 1423-4.

26. Nuffield Council on Bioethics. Briefing Note. Zika: ethical considerations. 9 Feb. 2016. Disponible en: http:// nuffieldbioethics.org/wp-content/uploads/NCOB-briefing-note-zika-ethical-considerations.pdf.

27. Lanini S, Zumla A, Ioannidis JP, Di Caro A, Krishna
S, Gostin L, et al. Are adaptive randomised trials or non-randomised studies the best way to address the Ebola outbreak in west Africa? Lancet Infect Dis 2015; 15: 738-45.

28. Basarab M, Bowman C, Aarons EJ, Cropley I. Zika virus. BMJ. 2016 Feb 26; 352:i1049. doi: 10.1136/bmj.i1049.

29. Moon S, Sridhar D, Pate MA, Jha AK, Clinton C, Delaunay $S$, et al. Will Ebola change the game? Ten essential reforms before the next pandemic. The report of the Harvard-LSHTM Independent Panel on the Global Response to Ebola. Lancet 2015; 386: 2204-21. 